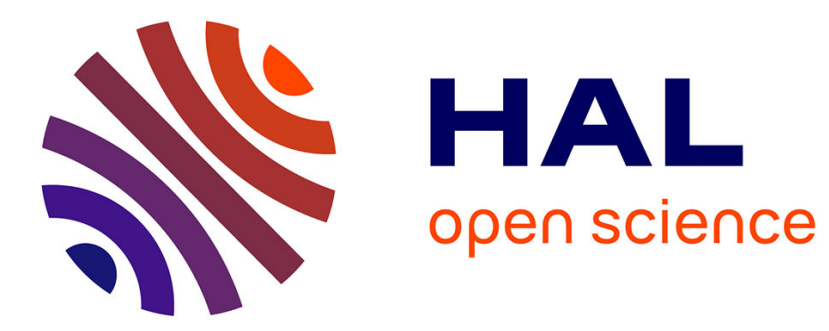

\title{
Une épée romaine découverte à Saintes (Charente-Maritime)
}

Nicolas Thomas, Michel Feugère, Nadine Dieudonné-Glad

\section{To cite this version:}

Nicolas Thomas, Michel Feugère, Nadine Dieudonné-Glad. Une épée romaine découverte à Saintes (Charente-Maritime). Gallia - Archéologie de la France antique, 2001, 58, pp.261-269. 10.3406/galia.2001.3027 . hal-00662339

\section{HAL Id: hal-00662339 \\ https://hal.science/hal-00662339}

Submitted on 23 Jan 2012

HAL is a multi-disciplinary open access archive for the deposit and dissemination of scientific research documents, whether they are published or not. The documents may come from teaching and research institutions in France or abroad, or from public or private research centers.
L'archive ouverte pluridisciplinaire HAL, est destinée au dépôt et à la diffusion de documents scientifiques de niveau recherche, publiés ou non, émanant des établissements d'enseignement et de recherche français ou étrangers, des laboratoires publics ou privés.

\section{(ㅇ)(1) $\$$}

Distributed under a Creative Commons Attribution - NonCommercial - NoDerivatives| 4.0 


\author{
Nicolas Thomas \\ Michel Feugère \\ Nadine Dieudonné-Glad
}

\title{
Une épée romaine découverte à Saintes (Charente-Maritime)
}

In: Gallia. Tome 58, 2001. pp. 261-269.

\section{Résumé}

Une épée longue, découverte à Saintes dans un contexte du lle s. de notre ère, peut être identifiée comme un rare exemple de spatha romaine, l'épée de cavalerie adoptée ultérieurement par l'infanterie à la place du gladius. Pourtant, l'analyse métallographique ne révèle aucun traitement particulier des tranchants qui permettrait de distinguer cette épée, utilisée pour frapper de taille, des glaives frappant d'estoc. L'arme, peut-être arrivée là où on l'a retrouvée pour être réparée ou transformée, permet donc de poser la question de la fabrication de ces spathae précoces et de leur fonction réelle dans l'équipement de l'armée romaine.

\section{Abstract}

A long sword found at Saintes in a context dated from the 2nd century AC, can be considered as a rare example of a Roman spatha, the cavalry sword which later on superseded the gladius in the infantry units. And yet, the metallographic analysis doesn 't show any peculiar treatment of the edges which could allow to distinguish this sword being used to cut, from the short sword used for sharp thrusting. The weapon may have arrived here for repairs and modifications and this raises questions about the production of these early spathae and their actual function in the Roman military equipment.

Citer ce document / Cite this document :

Thomas Nicolas, Feugère Michel, Dieudonné-Glad Nadine. Une épée romaine découverte à Saintes (Charente-Maritime). In: Gallia. Tome 58, 2001. pp. 261-269.

doi : 10.3406/galia.2001.3027

http://www.persee.fr/web/revues/home/prescript/article/galia_0016-4119_2001_num_58_1_3027 


\title{
UNE ÉPÉE ROMAINE DÉCOUVERTE À SAINTES (Charente-Maritime)
}

\author{
Nicolas THOMAS", Michel FeugÈRE* ${ }^{* *}$, Nadine DieudONNÉ-GLAD ${ }^{* * *}$
}

Mots-clés. Saintes, épée, cavalerie, spatha, militaria.

Key-words. Saintes, sword, cavalry, spatha, militaria.

Résumé. Une épée longue, découverte à Saintes dans un contexte du II s. de notre ère, peut être identifiée comme un rare exemple de spatha romaine, l'épée de cavalerie adoptée ultérieurement par l'infanterie à la place du gladius. Pourtant, l'analyse métallographique ne révèle aucun traitement particulier des tranchants qui permettrait de distinguer cette épée, utilisée pour frapper de taille, des glaives frappant d'estoc. L'arme, peut-être arrivée là où on l'a retrouvée pour être réparée ou transformée, permet donc de poser la question de la fabrication de ces spathae précoces et de leur fonction réelle dans l'équipement de l'armée romaine.

\begin{abstract}
A long stuord found at Saintes in a context dated from the $2^{\text {nd }}$ century $A C$, can be considered as a rare example of a Roman spatha, the cavalry sword which later on superseded the gladius in the infantry units. And yet, the metallographic analysis doesn't show any peculiar treatment of the edges which could allow to distinguish this sword being used to cut, from the short sword used for sharp thrusting. The weapon may have arrived here for repairs and modifications and this raises questions about the production of these early spathae and their actual function in the Roman military equipment.
\end{abstract}

\section{LE CONTEXTE DE LA DÉCOUVERTE}

La construction en 1999 de trois petites maisons à usage d'habitation, rue Montlouis à Saintes, a entraîné la découverte d'une épée dans un niveau d'abandon daté $\mathrm{du} \mathrm{II}{ }^{\mathrm{e}} \mathrm{s}$. de notre ère. Compte tenu de la surface modeste des parcelles concernées et de l'impact jugé faible sur les possibles vestiges, l'opération s'est limitée dans un premier temps à la surveillance du terrassement de tranchées de fondation très étroites $(50 \mathrm{~cm})$. La découverte de nombreuses structures à la limite du fond de fouille de l'aménageur a motivé quelques sondages com- plémentaires permettant une meilleure compréhension du site ${ }^{1}$.

Les parcelles sont situées au nord-ouest de Saintes, sur un plateau calcaire (Santonien) recouvert d'argile rouge à silex correspondant aux alluvions anciennes de la Charente. Le terrain présente un pendage sensible du nord vers le sud, révélant la présence d'un paléothalweg orienté est-ouest à l'emplacement de l'actuelle rue A.-Brunaud.

Traditionnellement, ce secteur est considéré comme étant situé à la limite de la ville du Haut-Empire. Des observations anciennes et de nombreuses fouilles

\footnotetext{
* AFAN, 13 boulevard Félix-Faure, F-93200 Saint-Denis.

** UMR 154 du CNRS, 390 avenue de Pérols, F-34970 Lattes.

*** UMR 6589 du CNRS (CESCM), Faculté des sciences humaines, 8 rue René-Descartes, F-86022 Poitiers cedex.

1. Financés en partie par le Service régional de l'archéologie de Poitou-Charentes.
} 


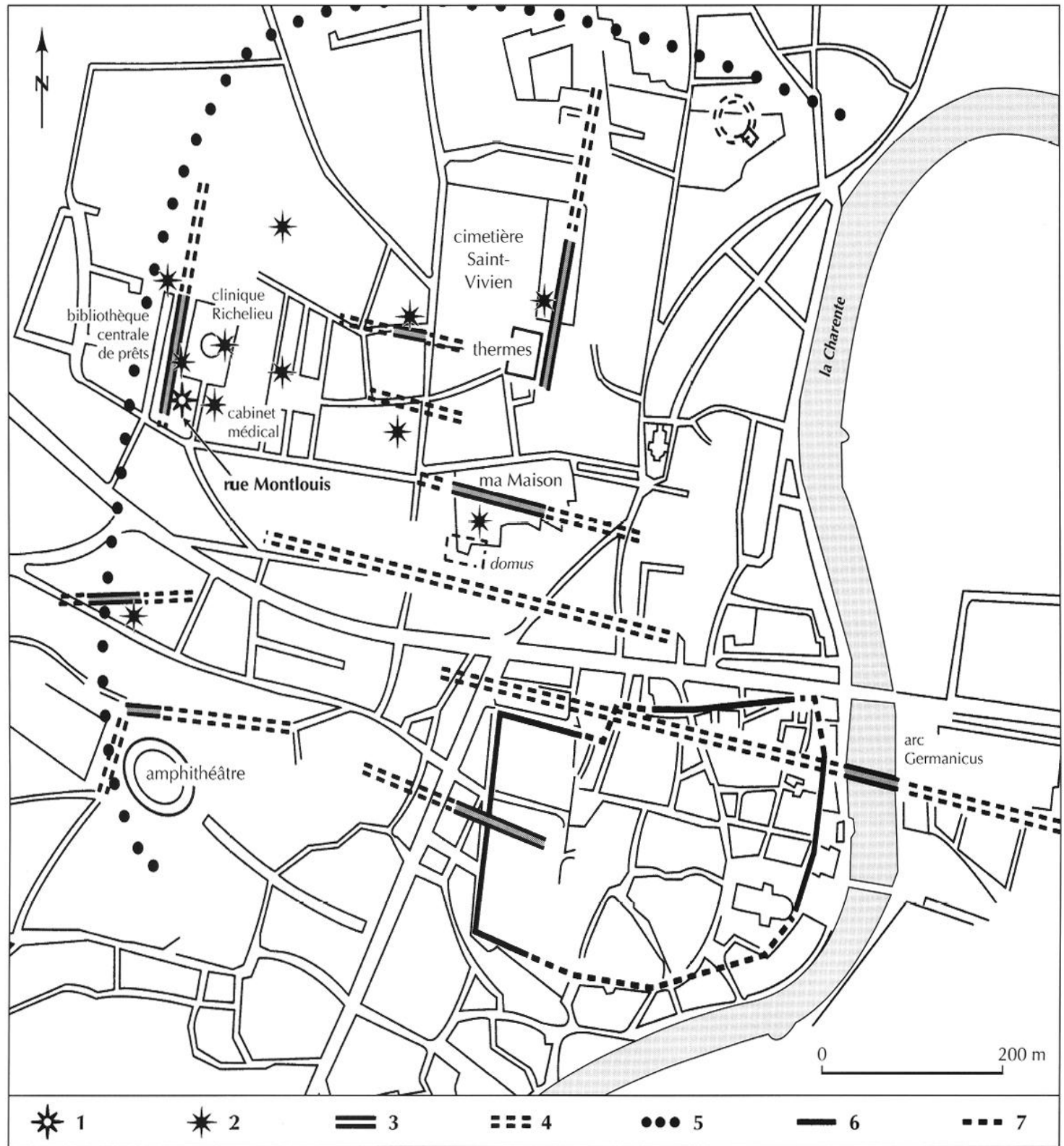

Fig. 1 - Saintes antique el localisalion du site : 1, site de la rue Montlouis ; 2, principaux siles à proximilé; 3, voie antique reconnue; 4, voie antique probable; 5, extension probable de la ville du Ilaut-Empire; 6, 7, rempart du Bas-Lmpire, sections reconnues et probables (fond de plan, d'après Maurin et al., 1994, p. 40).

récentes ont permis d'y localiscr des occupations du I ${ }^{\mathrm{cr}}$ et du II's. de notre ère (fig. 1). L'habitat antique semble diffus, et de nombreuses activités artisanales augustotibériennes ont été identifiées dans un rayon de quelques centaines de mètres seulement de la rue Montlouis. Nous pouvons rapidement mentionner la présence de fours de potier et d'un atelier de verrier (Dangibcaud, 1933-1935 ; Hillairet, 1990, p. 89 ; Buisson, 1994). Plus près, sur les parcelles mitoyennes de l'intervention, de nombreuses traces ténues d'activités métallurgiques telles que des scories de forge ont été repérées sur les sites de la Clinique Richclicu (au nord), du Cabinet médical (à l'ouest) et de la Bibliothèque centrale de prêt (à l'est) ${ }^{2}$.

2. Pour la Clinique Richelieu : Bégué et al., 1972 ; Hillairet, 1993 ; Robin, 1993 ; Baigl, 1995 ; Bocquet, Jourde, 1996 ; pour le Cabinet médical : Doyen, 1992 ; pour la Bibliothèque centrale de prêt : Vernou, 1989. 
Malgré l'exiguité des sondages, nous avons mis en évidence deux ensembles bâtis distincts et probablement érigés au cours de la première moitié du ${ }^{\mathrm{er}} \mathrm{s}$. Les deux bâtiments semblent construits en terrasse le long d'une pente naturelle et sont sans doute à mettre en relation avec l'aménagement du quartier sous Tibère, comme l'indiquent les fouilles réalisées dans les environs immédiats. L'hypothèse est confirmée à la fois par les éléments de datation recueillis et par l'orientation des structures linéaires. Les différentes constructions s'intègrent dans un schéma directeur plus large autour d'une trame viaire en partie connue ${ }^{3}$. Après quelques réaménagements mineurs, le site paraît très vite abandonné aux alentours du milieu du II $^{c}$ s., rejoignant ainsi les diverses observations réalisées dans ce secteur. Enfin, une inhumation en cercueil cloué, mais difficilement datable du fait de l'indigence du mobilier associé, vient couper les derniers niveaux d'abandon du site.

Dans les deux zones observées, le mobilier archéologique est relativement abondant (fig. 2). Outre quelques intrusions facilement explicables par la proximité de la surface et par les méthodes de terrassement, deux ensembles homogènes peuvent être reconnus. Le premier, correspondant à l'aménagement et à l'occupation du site, suggère une datation aux alentours de la première moitié du ${ }^{\mathrm{er}} \mathrm{s}$. Nous pouvons mentionner la présence de céramique noire savonneuse, d'une amphore Pascual 1 ou encore d'une fibule en bronze de type F. 22d (Feugère, 1985, p. 312-324). Le second ensemble a été recueilli dans les niveaux d'abandon du $\mathrm{II}^{\mathrm{e}}$ s. Nous avons rencontré dans ces niveaux quelques éléments caractéristiques, comme une bague en bronze avec de petits cabochons probablement émaillés à l'origine (ou incrustations de verre sur les côtés), dont le type est bien attesté au II ${ }^{\mathrm{e}} \mathrm{s} .{ }^{4}$, et une monnaie gauloise très répandue dans la région au $\mathrm{I}^{\mathrm{er}} \mathrm{s}$. Le dernier niveau de comblement d'un puits indique cependant une sédimentation postérieure aux années 70

3. La fouille à l'emplacement de l'actuelle Bibliothèque centrale a permis de mettre au jour une voie orientée nord-sud et bordée d'un portique (Vernou, 1989). Une autre voie perpendiculaire a été repérée en coupe lors de l'intervention sur le site du Cabinet médical (Doyen, 1992). Voir également Hillairet (1996) et Thomas (à paraître).

4. Pour le type, $c f$. Henkel, 1913 , $\mathrm{n}^{\text {(* }} 1083$ et $1333-4$; Riha, 1990, p. 33 et pl. 7, $\mathrm{n}^{\text {\%s }}$ 108-110 (un seul cabochon circulaire émaillé du II ${ }^{\mathrm{c}}$ s.). Il s'agit ici de la forme précoce d'un modèle qui évolue ensuite, au III' $s$., vers des épaulements plastiques plus marqués.
Illustration non autorisée à la diffusion

Fig. 2 - Petit mobilier en bronze: 1, fibule F. 22d, niveau d'occupation du $I^{e r}$ s. ; 2, applique militaire, niveaux d'abandon du II s. ; 3, bague, niveau associé à l'épée (dessins F. Blanchet).

à 120 , notamment grâce à la présence de céramique sigillée Drag. 36 et 37 produite à Montans ${ }^{5}$. L'épée en fer, appartenant au second ensemble, a été découverte au sommet d'un mur arasé dont les matériaux avaient fait l'objet d'une récupération partielle. Outre la bague en bronze déjà signalée, le mobilier datant associé à l'épée

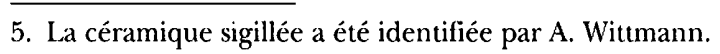


est représenté par de la céramique (Drag. 35 avec décor en feuille d'eau), mais surtout par de la céramique à paroi fine et engobe marbré attestée à Saintes à partir de la seconde moitié du II $^{e} \mathrm{~s}$. Le reste de la céramique commune (grise) est également représentatif de cette période.

En l'état de nos connaissances, la nature de l'occupation est difficile à préciser. Dans presque toutes les unités stratigraphiques, nous avons trouvé de nombreuses scories de fer. Elles sont présentes à la fois dans les niveaux d'occupation et dans les couches d'abandon du site. Nous avons identifié des parois de four en argile cuite, avec des résidus scoriacés adhérant, des scories spongicuses et des scories en calotte, toutes deux caractéristiques d'une forge. L'une de ces scories présente un appendice typique; elle a été trouvée dans un niveau compact, composé exclusivement de résidus scoriacés, le tout sur un sol en calcaire pilé et rubéfié. Toutefois, l'étude des prélèvements de sédiment effectués dans la plupart des niveaux rencontrés n'a pas permis de repérer de battiture. Nous ne pouvons donc pas confirmer la présence d'une forge sur le site, même si de nombreux indices semblent attester la présence d'une activité métallurgique dans le quartier.

\section{L'ÉPÉE}

L'épée a été retrouvée dans un état de conservation remarquable (fig. 3) ; elle n'a souffert que d'une légère pliure, à mi-longueur, apparemment due à la pression des pierres contre lesquelles elle s'est trouvée bloquée au moment de son enfouissement. La soie, longue de $20 \mathrm{~cm}$, semble complète ; elle est de section rectangulaire légèrement croissante vers la garde. Pommeau, poignée et garde pouvaient être en bois (on n'en a retrouvé aucune trace). La lame, dont le sommet forme deux angles droits très marqués, a une largeur maximale de $45 \mathrm{~mm}$ : les bords en sont presque parallèles et on n'observe qu'un rétrécissement très progressif, puisque la largeur est encore de $32 \mathrm{~mm}$ à $5 \mathrm{~cm}$ de la pointe, qui est de forme ogivale. Avec sa section losangique et sa longueur totale de $825 \mathrm{~mm}$, cette épée présente donc toutes les caractéristiques d'une arme romaine du début de l'Empire. On reconnaît l'épée longue, ancêtre de la spatha, utilisée au $\mathrm{I}^{\mathrm{er}}$ s. par la cavalerie avant d'être adoptée, au III' ${ }^{\mathrm{s}}$., par l'ensemble de l'infanterie romaine.

\section{Illustration non autorisée à la diffusion}

Fig. 3 - Épée romaine de Saintes (cliché A. Dagorn, Invenlaire général, 1999, (C ADAGP; dessin F: Blanchet).

Les découvertes d'armes de ce type sont d'une très grande rareté. Cette situation est aggravée sans doute par le fait que ces épées n'ont pas fait l'objet des inventaires européens dont ont pu bénéficier, par ailleurs, les glaives, poignards ou casques romains. On en est donc réduit à citer quelques exemples, qui permettront de replacer la 
découverte de Saintes dans son contexte. En Gaule, une seule trouvaille avait été signalée jusqu'à ce jour: une épée recueillie dans le Doubs à Pontoux, conservée au musée de Chalon-sur-Saône sous le $\mathrm{n}^{\circ}$ 69.1.60 (Bailly, $1977, \mathrm{n}^{\circ} 10$; Feugère, 1990, $\mathrm{n}^{\circ} 112$, fig. 89). Longue de $840 \mathrm{~mm}$, elle se présente sous une forme très proche de l'arme saintaise : taille voisine, soie allongée, lame à bords parallèles semblant avoir été rendus légèrement concaves par des affûtages répétés, section losangique, pointe ogivale renforcée ici par un léger épaississement, comme sur certains glaives du $\mathrm{I}^{\mathrm{er}} \mathrm{s}$. Bien que découverte hors contexte, cette épée a été considérée comme une arme de cavalerie par comparaison avec l'épée de Rottweil.

Cette dernière, qui représente à ce jour la seule épée de ce type au nord des Alpes, est moins bien conservée que les armes de Saintes ou de Pontoux (Planck, 1975, pl. 79, 3 ) ; atteignant une longueur de $1020 \mathrm{~mm}$, elle montre une lame pratiquement identique aux précédentes. Elle a été découverte en 1883 ou 1884 dans un secteur de la ville situé à l'est du Neckar ("Hochmauren ») où sont localisés les vestiges de plusieurs camps. Sur la rive occidentale du Neckar, on connaît par ailleurs les restes d'un camp qui a abrité une partie de la légion XI Claudia, ainsi que des auxiliaires (Sommer, 1992, p. 270). La partie de la légion XI Claudia qui a été stationnée à Rottweil a pu l'être durant une période plus brève qu'à Vindonissa: 73/74 - ca. 90 (Francke, 1997, p. 31), alors que de leur côté les auxiliaires ont pu rester jusqu'au début du $\mathrm{II}^{\mathrm{e}} \mathrm{s}$. de notre ère (Sommer, 1992, p. 272 sqq.). Parmi les tuiles estampillées de Rottweil, on ne connait cependant aucune aile de cavalerie, seulement des cohortes. L'épée de Rottweil a donc pu appartenir à un soldat d'une cohorte mixte, d'autant plus que l'on connaît à Rottweil une cohorte II Aquitanorum equitata civium Romanorum (Schönberger, 1985, p. 455) qui permet d'établir un autre lien avec le sud-ouest de la Gaule ${ }^{6}$.

On ne peut cependant passer sous silence, puisqu'elle est attestée à Saintes même, l'existence de la cohorte $I$ Belgarum, equitata à partir des Flaviens (ce qui correspond parfaitement à notre contexte), dont le célèbre Santon C. Iulius Victor a été tribun (ILA, 18, cf. Maurin et al., 1994). Force est de constater qu'en-dehors du dénominateur commun fourni par la ville de Saintes, où le tribun eut son mausolée, aucun lien direct ne peut être

6. Nous remercions M. Luik (université de Trier) pour l'aide apportée à cet aspect de l'étude.

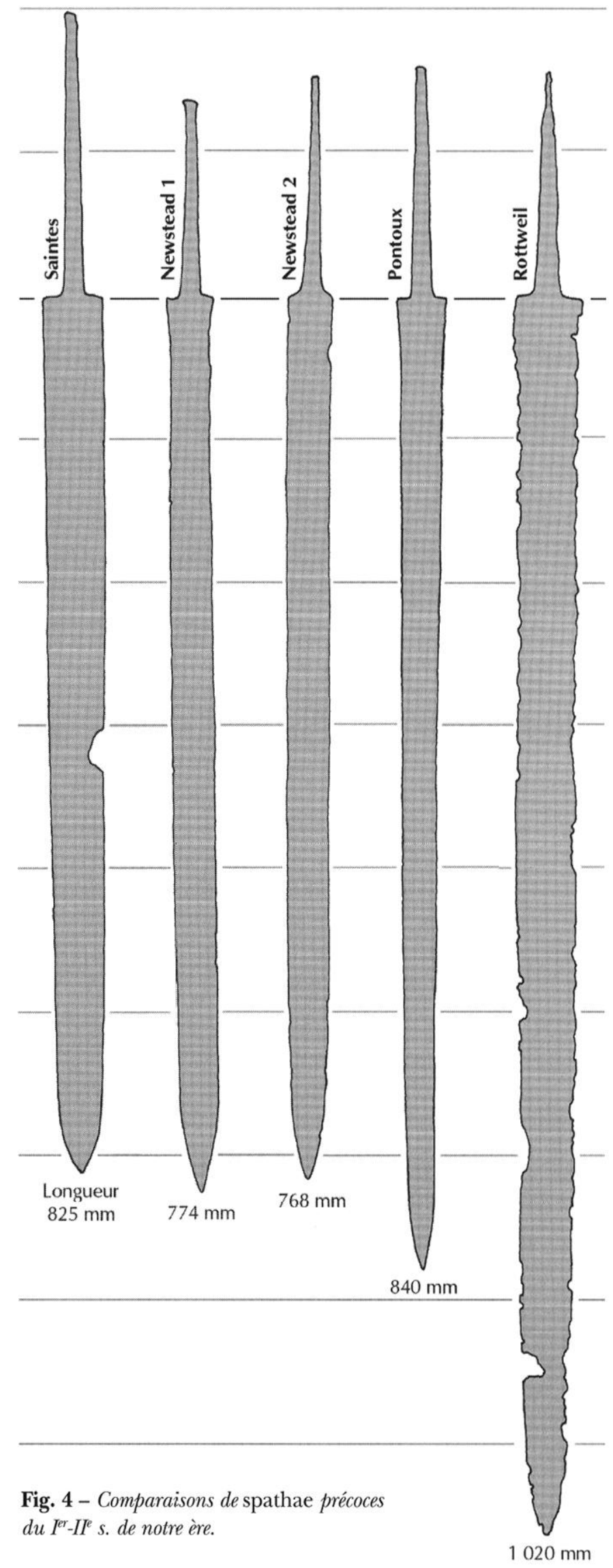




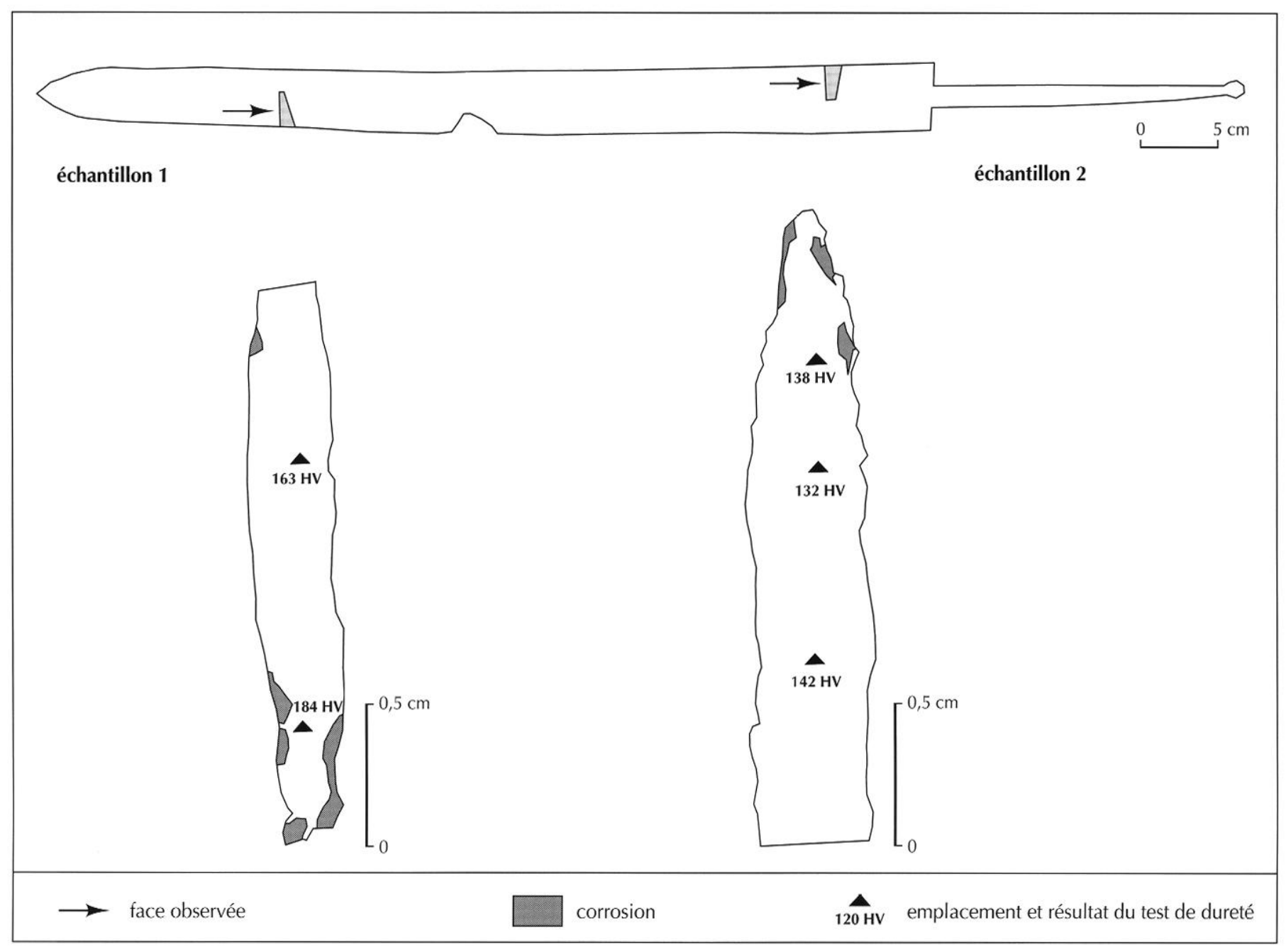

Fig. 5 - Schéma des prélèvements et résultats des lests de dureté effectués sur l'épée.

établi entre la découverte de l'épée de Saintes ct ce corps de troupes.

Enfin, deux autres spathae précoces (fin $\mathrm{I}^{\mathrm{er}}$ et $\mathrm{II}^{\mathrm{e}} \mathrm{s}$. de notre ère) ont été recueillies au début du siècle dans le camp de Newstead (Curle, 1911, pl. XXXIV, 6 et 7). Ces épées très proches l'une de l'autre (longueurs 774 et $768 \mathrm{~mm}$ ) présentent beaucoup de similitudes avec l'épée de Pontoux, mais restent nettement plus étroites que la spatha de Saintes. La comparaison des cinq épées romaines précoces évoquées ici, asse\% différentes dans leurs proportions (fig. 4), suffit à mettre en évidence les relations étroites qui unissent les épées de Newstead et de Pontoux d'une part, les armes de Saintes et Rottweil d'autre part; la longueur de cette dernière dépasse néanmoins largement celle de toutes les autres.
Tout en illustrant la variabilité d'épées romaines plus ou moins contemporaines, ce rapide survol met donc en évidence les liens morphologiques entre deux des trouvailles, Saintes et Rottweil. Il est remarquable que ce rapprochement puisse être éventuellement mis en relation avec un possible déplacement de troupes originaires d'Aquitaine.

\section{EXAMEN MÉTALLOGRAPHIQUE}

Au-delà de son intérêt typologique, il paraît utile d'étudier cet objet d'un point de vue structural, notamment dans la perspective d'une comparaison avec plusicurs épées de type gladius provenant du lit de la Saône et récemment publiées (Dieudonné-Glad, Parisot, 1998). En effet les gladii sont des armes d'estoc dont la 
pointe est active, alors que les spathae sont des armes de taille dont toute la longueur du tranchant est sollicitée. Un des objectifs de l'étude structurale est donc d'observer si cette utilisation différente est concomitante à des techniques de fabrication particulières. On s'attendrait, par exemple, à un traitement du tranchant de la spatha afin de le rendre plus efficace.

Deux prélèvements ont été effectués sur la lame de l'épée ${ }^{7}$, chacun sur un tranchant. Les échantillons ont la longueur d'une demi-section de la lame et ont été observés sur une de leur face (fig. 5) après enrobage de résine, polissage et attaque au nital (solution de $5 \%$ d'acide nitrique dans de l'éthanol).

L'épée est constituée d'un métal très peu carburé (fig. 6) contenant d'assez nombreux îlots d'inclusions de scories (fig. 7), témoins d'une épuration médiocre du métal issu de la loupe. La présence de chapelets d'inclusions aplaties témoigne probablement d'une préparation du métal par repli sur lui-même. Sur chacun des échantillons, un des plats de l'épée comporte une zone légèrement carburée, à structure perlitique, mais sur l'échantillon 1 il s'agit du plat supérieur, tandis que sur l'échantillon 2, la partie carburée se situe sur le plat inférieur. Ces zones légèrement carburées ne correspondent donc probablement pas à une utilisation volontaire d'acier, mais sont la conséquence de l'hétérogénéité initiale de la loupe de fer. D'ailleurs, si la partie carburée de la lame se prolonge jusqu'au tranchant de l'échantillon 1, ce n'est pas le cas pour l'échantillon 2. En fait, l'essentiel du métal est de la ferrite dont les grains sont équiaxes et de grande taille.

L'observation structurale a été complétée par des essais de dureté Vickers (fig. 5). La dureté se répartit entre 132 et $163 \mathrm{HV}$ dans les zones ferritiques et est de $184 \mathrm{HV}$ pour une des zones légèrement carburées. Cette dureté reste donc partout très faible.

Cette épée a donc été fabriquée à partir d'une loupe dc mćtal obtcnue par réduction directe du minerai de fer. Celle-ci était inégalement carburée, avec des taux de carbone très faibles. L'objet est constitué essentiellement de fer doux, de faible dureté, et n'a subi aucun traitement visant à améliorer les performances de ses tranchants, comme la soudure d'un tranchant aciéré ou un durcisse-

7. Nous remercions J. Parisot (LMPM, ENSMA, Poitiers) qui a veillé personnellement à la bonne exécution des prélèvements. L'étude métallographique a également été effectuée au Iaboratoire de métallurgie et de physique des matériaux de l'École nationale supérieure de mécanique et d'aérotechnique à Poitiers. ment par martelage à froid. La lame devait donc être particulièrement ductile et son tranchant facilement déformable, ce qui la rendait peu apte à l'usage auquel elle était

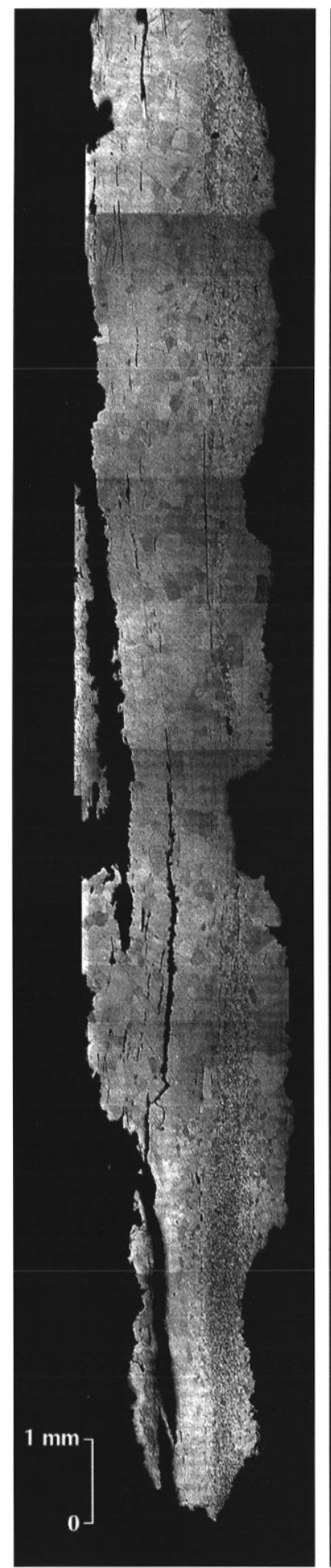

échantillon 1

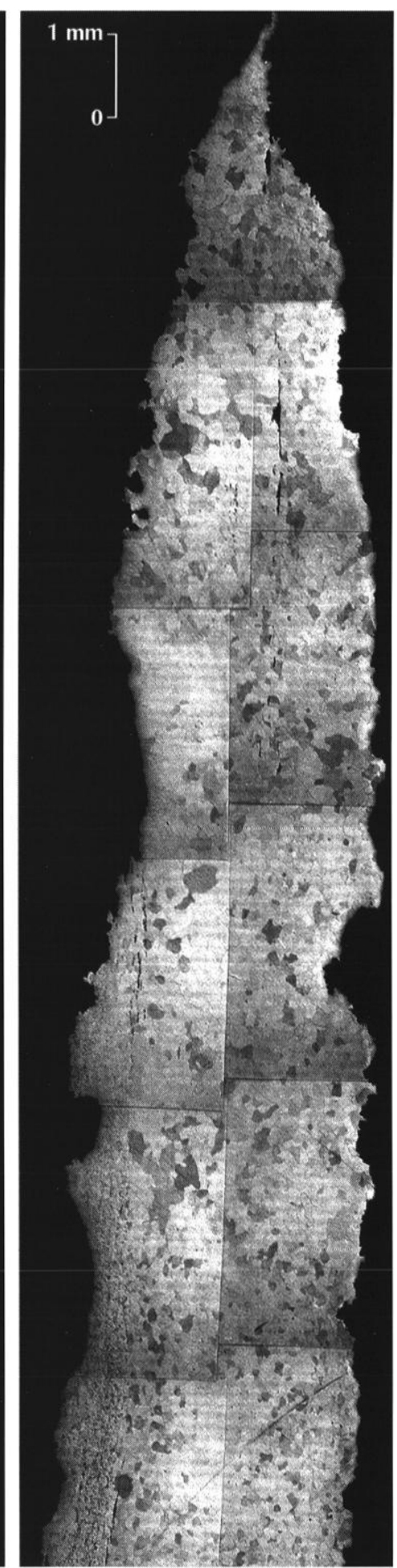

échantillon 2
Fig. 6 - Métallographie des échantillons prélevés sur l'épée. 


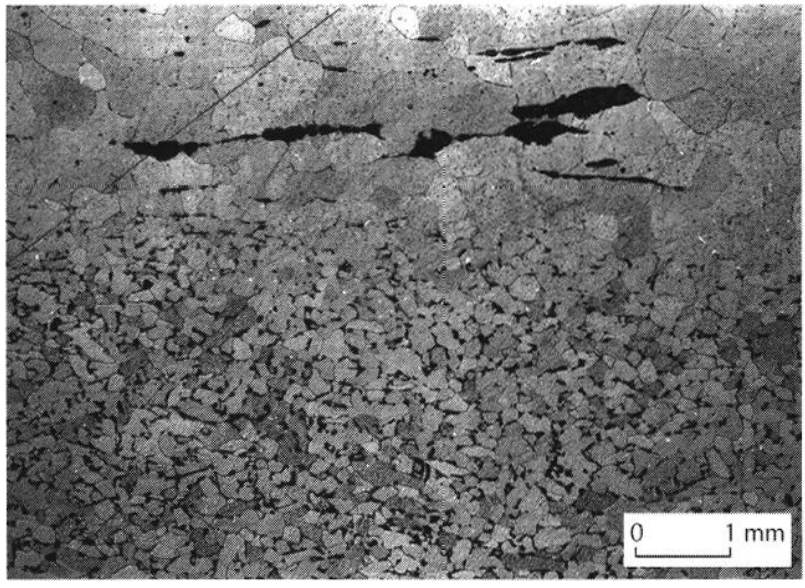

Fig. 7 - Détail de l'échantillon 1 de l'épée: zone ferritique en haut, zone faiblement carburée en bas, inclusions de scories en sombre.

destinée. Si on la compare aux gladii du musée Denon à Chalon-sur-Saône, on ne peut mettre en évidence aucun procédé particulier appliqué aux tranchants de l'épée de Saintes dans l'intention de les rendre plus durs. D'autre part, la qualité du métal employé est plutôt plus médiocre que celle de deux des gladii de Chalon, fabriqués avec un acier à teneur en carbone assez uniforme permettant d'obtenir des duretés moyennes sensiblement supérieures à celles observées sur la spatha de Saintes.
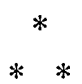

La découverte à Saintes d'une épée longue, identifiée comme spatha de cavalerie du I ${ }^{\mathrm{er}}$ ou du début du $\mathrm{II}^{\mathrm{e}} \mathrm{s}$. de notre ère, intéresse deux domaines distincts de la recherche :

- d'une part, l'exploration archéologique de la ville de Saintes, où plusieurs indices permettent de supposer la présence, sans doute épisodique, de militaires stationnés dans la région ou de passage ; sur la base du rapprochement avec Rottweil, on peut se demander si le propriétaire de cette épée n'était pas un cavalier auxiliaire, membre par exemple de la cohorte II Aquitanorum equitata civium Romanorum;

- d'autre part, l'étude des armes romaines, au sein desquelles les premières spathae sont encore particulièrement mal connues; de ce point de vue, au-delà des mensurations pouvant indiquer une certaine standardisation de ces épées longues, l'étude métallographique révèle que les tranchants n'ont pas fait l'objet d'un traitement particulier. Pour surprenant qu'il puisse paraitre pour une arme destinée à frapper de taille et non d'estoc, ce résultat est peut-être à mettre en relation avec une origine marginale (initiatives privées ? réactivation d'une technologie abandonnée en Gaule depuis plus d'un siècle ?) des épées longues de cavalerie, dont beaucoup peuvent avoir été produites au sein des groupes ethniques constituant la cavalerie romaine aux $\mathrm{I}^{\text {er }}$ et $\mathrm{II}^{\mathrm{c}} \mathrm{s}$. de notre ère. 


\section{BIBLIOGRAPHIE}

BAIGL J.-P.

1995 : Saintes, Montlouis, Clinique Richelieu, Rapport d'expertise archéologique, Poitiers, $17 \mathrm{p}$.

BAILly A.

1977 : Les armes d'époque romaine dans la vallée de la Saône, Mémoires de la Société historique et archéologique de Chalon-sur-Saône, 47, p. 159-172.

BÉGuÉ J.-P., TIllhard J.-L., Rouvreau M. 1972 : La céramique gallo-romaine recueillie à la Clinique Richelieu à Saintes, Revue de la Saintonge et de l'Aunis, XLVIII, $7^{\mathrm{c}}$ fasc., p. 4-21.

BoCQUET A., JOURDE E.

1996 : Saintes, Clinique Richelieu, Document final de synthèse, Poitiers, $14 \mathrm{p}$.

BuISSON J.-F.

1994 : Montlouis : un nouvel atelier de potier au nord de Saintes dans l'Antiquité, Bulletin de la Société d'archéologie et d'histoire de la CharenteMaritime, 20, p. 109-111.

CURLE J.

1911 : A Roman Frontier Post and its People: the Fort of Newstead in the Parish of Melrose, Glasgow, James Maclehose \& Sons.

Dangibeaud C.

1933-1935 : Montlouis : un nouvel atelier de potier au nord de Saintes dans l'Antiquité, Bulletin de la Société des archives historiques de la Saintonge et de l'Aunis, XLV, p. 131.

Dieudonné-Glad N., Parisot J.

1998 : Étude métallographique d'épées celtiques et romaines du musée Denon à Chalon-sur-Saône, in : Nicolini G., Dieudonné-Glad N. (éds), Les métaux dans l'Antiquité, Actes du colloque de Poitiers, 25-28 sept. 1995, Monographie Instrumentum, 6, Montagnac, p. 153-163.

DOYEN D.

1992 : Site du Cabinet médical, 22 rue
Montlouis, Recherches archéologiques en Saintonge 1992, Saintes, Publication de la Société d'archéologie et d'histoire de la Charente-Maritime, p. 35-54.

\section{FEUGÈre M.}

1985 : Les fibules en Gaule méridionale, Paris, éd. du CNRS, 509 p., 174 pl.

1990 : Les armes romaines, in: Bonnamour L. (dir.), Du silex à la poudre, 4000 ans d'armement en val de Saône, Montagnac, éd. Monique Mergoil, p. 92-115.

1993 : Les armes des Romains, de la République à l'Antiquité tardive, Paris, éd. Errance, 287 p.

1996 : L'armement du Bas-Empire, in: Reddé M. (dir.), L'armée romaine en Gaule, Paris, éd. Errance, p. 267-278.

\section{FRANCKE R}

1997 : Ein Lager der legio XI Claudia in Arae Flaviae/Rottweil und die Besetzung der oberen Neckargebietes, Gesellschaft Pro Vindonissa, p. 25-32.

HENKEL F.

1913 : Die römischen Fingerringe der Rheinlande und der benachbarten Gebiete, Berlin, Georg Reiner.

Hillairet J.-L.

1990 : Puits d'un verrier antique au 85 rue de la Boule, Bulletin de la Sociêté d'archéologie et d'histoire de la CharenteMaritime, 17, p. 89-93.

1992 : Sondage d'un terrain au 85 rue de la Boule à Saintes en 1988, Recherches archéologiques en Saintonge 1992, Saintes, Publication de la Société d'archéologie et d'histoire de la Charente-Maritime, p. 55-68.

1993 : Clinique Richelieu 1992, fouille de sauvetage urgent, Rapport de fouille, Saintes, Publication de la Société d'archéologie et d'histoire de la Charente-Maritime, n.p., 29 pl.

1996 : Synthèse des connaissances sur la voirie antique de Saintes, essai de restitution du réseau, Recherches archéologiques en Saintonge 1994, Saintes,
Publication de la Société d'archéologie et d'histoire de la CharenteMaritime, p. 85-106.

Maurin L., Thauré M., Tassaux F.

1994 : Inscriptions latines d'Aquitaine (ILA), Santons, Bordeaux, Centre PierreParis, 518 p.

PlanCK D.

1975 : Arae Flaviae I, Neue Untersuchungen zur Geschichte des römischen Rottweil, Stuttgart, Theiss.

RiHA E.

1990 : Der römische Schmuck aus Augst und Kaiseraugst, Forschungen in Augst, 10, Augst.

RoBIN K.

1993 : Montlouis, Clinique Richelieu, Sauvetage urgent, Document final de synthèse, $85 \mathrm{p}$.

SCHÖNBERGER H.

1985 : Die römischen Truppenlager der frühen und mittleren Kaiserzeit zwischen Nordsee und Inn., Bericht der Römisch-Germanischen Kommission, 66 , p. 321-497.

SOMMER C. S.

1992 : Municipium Arae Flaviae : militärisches und ziviles Zentrum im rechtsrheinischen Obergermanien, Bericht der Römisch-Germanischen Kommission, 73, p. 269-313.

THOMAS N.

À paraître : Une voie du Haut-Empire à Saintes, allée de la Poudrière, Recherches archéologiques en Saintonge 1998, Saintes, Publication de la Société d'archéologie et d'histoire de la Charente-Maritime.

VERNOU C.

1989 : Le site gallo-romain de la Bibliothèque centrale de prêt à Saintes, Revue de la Saintonge et de l'Aunis, XV, p. 7-54. 